\section{THEORETICAL RESEARCH ON THE GAS PHASE DENSITY CHANGE IN PROCESSES OCCURRING DURING WORK OF THE TRANSPORT MODULE INTENDED FOR TRANSPORT FROM THE SEABED}

DOI 10.2478/ntpe-2018-0075

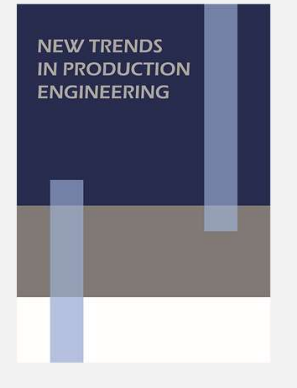

2018

Volume 1

Issue 1

pp. 597-604

Wiktor Filipek PhD.

Krzysztof Broda PhD.

AGH University of Science and Technology, Poland

\begin{abstract}
In recent years, we have observed a great interest in the exploitation of marine deposits by various methods of mining and transport to the surface. However, obtaining natural resources deposited at greater depths such as polymetallic nodules and seafloor massive sulphides - SMS creates a lot of challenges for both scientists and engineers. The solutions developed so far, unfortunately, have so far been characterized by high energy consumption.

For several years the authors have been conducting theoretical and experimental research on new concepts of seabed to surface transport. The results of them have been presented in previous publications. This publication presents the results of the continuation of research on the concept of construction and operation of an autonomous transport module (submitted for printing). It focuses on a theoretical analysis of the change in gas phase density in the processes occurring during operation of the transport module intended for transport from the seabed. For this purpose, a reduced form of the van der Waals equation was used in the form of a third-degree equation for parameters interested from the point of view of the transport module.
\end{abstract}

Keywords: Marine mining, transport from great depths, autonomous transport module

\title{
INTRODUCTION
}

Due to the depleting resources in land deposits of many minerals (especially critical elements), investors and researchers have been showing a growing interest in sea and ocean deposits. Shelf resources have been successfully exploited in many places on Earth using various methods (Karlic, 1984; Depowski et al., 1998; Sharma, 2017). However, due to the extreme conditions in these environments, exploiting deep-sea resources is posing significant difficulties for both scientists and engineers. Nevertheless, this does not discourage attempts to exploit these resources, as reflected in the increasing number of consortia involved in research into various methods of extraction and surface transport of manganese nodules and polymetallic massive sulphides (SMS) (SPC, 2013). Regrettably, these operations are, in most cases, highly energy-intensive.

The authors of this article have been conducting research into new sea-bottom transport concepts for the last several years. Their work has yielded a number of publications in the previous years and this year (Filipek and Broda, 2016, 2017). In these publications, we presented the results of theoretical studies related to the application of a new method in which pyrotechnic materials are used as a source of energy in sea-bottom transport, as well as experimental studies using potassium nitrate and ammonium nitrate in a controlled pyrotechnic reaction, and calcium carbide. We also compared three concepts of transporting output from the sea bottom in terms of the energy demand they entail. In the last publications, we presented a concept involving the construction of an autonomous transport module and experimentally confirmed its viability for sea-bottom transport using calcium carbide. The fundamental problem we have faced during our work on the new concepts of sea-bottom transport was related to determining the density of the analysed substances in the gas phase 
as a function of pressure and temperature. So far, we have been using tools available online (calculators of gas parameters under predefined conditions) and Standing-Katz charts (http://petrowiki.org/images/7/76/Vol1_Page_225_Image_0001.png;

https://pl.scribd.com/doc/41671999/Standing-Katz-Chart), and iteration methods. Using this calculation approach, we were able to determine selected gas-state parameters for specific conditions. Regrettably, this method was not suitable for a continuous simulation of the process. Such a full description of the process is particularly desirable for the understanding of how an autonomous transport module works - the principles of its operation are presented in the previous article (submitted for printing) and patent applications.

We have had experience with methods involving Standing-Katz chart curve fitting by various functions and with their application for the simulation of investigated processes. We claim that the Standing-Katz chart curves provide solutions to the reduced form of the Van der Waals equation. Unfortunately, we were unable to find any publication confirming this hypothesis, so in this article, we decided to present analytical solutions to the reduced Van der Waals (Berry et al., 2000; Smith, 2005; Xiang, 2005; Mizerski, 2013) equation for the parameters that are relevant for the operation of autonomous transport modules.

\section{CONVERTING THE VAN DER WAALS EQUATION TO A CUBIC EQUATION}

To derive the desirable form of the Van der Waals equation, let us start with the classic form of the Van der Waals equation (Berry et al., 2000; Smith et al., 2005; Xiang, 2005; Mizerski, 2013):

$$
\left(p+n^{2} \frac{a}{V^{2}}\right)(V-n b)=n R T
$$

The above equation usually provides a good approximation of the real-gas state equation. Regrettably, from our perspective, this equation is too complex for analytical solutions, since it is a function of as many as six parameters: the intermolecular attraction $\left[\mathrm{J} \cdot \mathrm{m}^{3} / \mathrm{mole}^{2}\right]$, the excluded volume $b\left[\mathrm{~m}^{3} / \mathrm{mole}\right]$, the gas amount $n$ [mole], the pressure $p[\mathrm{~Pa}]$, the volume $V\left[\mathrm{~m}^{3}\right]$ and the temperature $T[\mathrm{~K}]$.

By adding to the above equation the reduced values of the pressure $p_{Z}$, the temperature $T_{Z}$, the volume $V_{z}$ as defined below

$$
\begin{aligned}
p_{z} & =\frac{p}{p_{k r}} \\
T_{z} & =\frac{T}{T_{k r}} \\
V_{z} & =\frac{V}{V_{k r}}
\end{aligned}
$$

and substituting critical parameters (the pressure $p_{k r}$, the temperature $T_{k r}$, and the volume $V_{k r}$ ) with the known relationships (Xiang, 2005; Mizerski, 2013):

$$
\begin{gathered}
p_{k r}=\frac{1}{27} \frac{a}{b^{2}} \\
T_{k r}=\frac{8}{27 R} \frac{a}{b} \\
V_{k r}=3 b
\end{gathered}
$$

after certain conversions, we obtain a "reduced" form of the Van der Waals equation:

$$
\left(p_{z}+n^{2} \frac{3}{V_{z}^{2}}\right)\left(3 V_{z}-n\right)=8 n T_{z}
$$

The resulting equation has a universal form with four parameters: $n, p_{Z}, V_{z}$, allowing us to clearly determine the physical state of the gas.

The parameter we mentioned in the introduction which is of prime interest to us in the context of sea-bottom transport is the density $d\left[\mathrm{~kg} / \mathrm{m}^{3}\right]$ of the analysed gas as a function of pressure and temperature. Knowing $n$, the molar mass $M[\mathrm{~g} / \mathrm{ml}]$ and the volume $V$ of the analysed gas, we can determine its density

$$
d=0.001 \cdot n \frac{M}{V}
$$

Therefore, we look for a relationship which would allow us to determine the replacement density $V_{z}\left(n, p_{Z}, T_{z}\right)$, and, by extension, the volume $V(n, p, T)$. To this end, we convert equation (4) to the cubic equation form:

$$
3 p_{z} V_{z}^{3}-n\left(p_{z}+8 T_{z}\right) V_{z}^{2}+9 n^{2} V_{z}-3 n^{3}=0
$$


From a mathematical point of view, we know that each cubic equation has at least one root in the set of real numbers. To solve the above equation (6), we can employ Cardano's formulas (Nickalls, 1993; Bronsztejn and Siemiendiajew, 2000). According to these formulas, the solution to a cubic equation in the set of real numbers depends on the value of the "discriminant" $\Delta$. Thus, when

$\Delta>0$, then there is one real root

$\Delta=0$ then there are two real roots

$\Delta<0$ then there are three real roots

As presented graphically in Figure 1

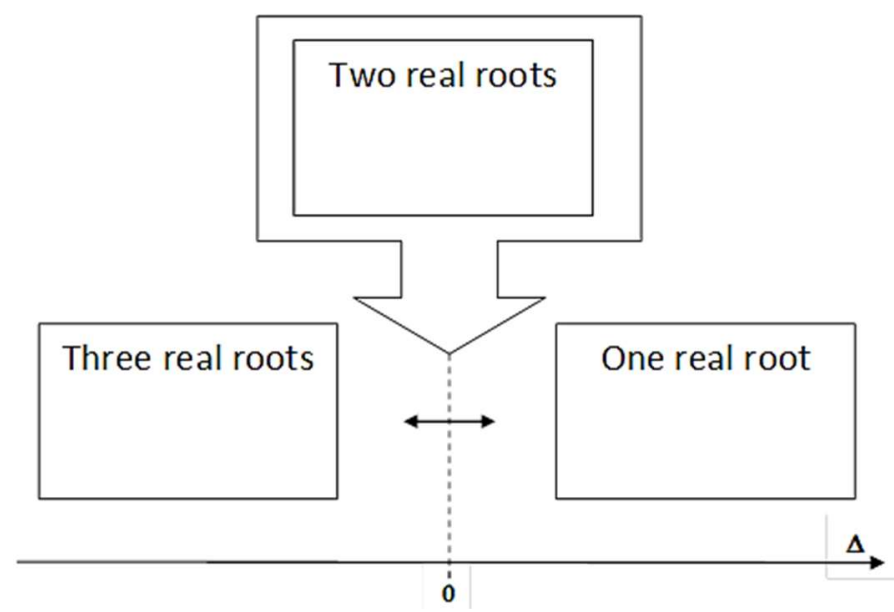

Fig.1 Graphic interpretation of the solution method.

\section{DETERMINING THE PHYSICAL ROOTS OF THE REDUCED VAN DER WAALS EQUATION OF STATE AS A CUBIC EQUATION}

To solve equation (6), we start by determining the "discriminant" $\Delta$ which is, in actuality, a function of two parameters: $p_{z}$ and $T_{z}$, as demonstrated below. Therefore, it was denoted as the parameter $\Delta_{p} T$ :

$$
\Delta_{p T}=\Delta_{p T}\left(n, p_{z}, T_{z}\right)=\frac{n^{6}}{729 p_{Z}^{4}} f\left(p_{Z}, T_{Z}\right)
$$

where:

$$
f\left(p_{Z}, T_{z}\right)=p_{z}^{3}+\left(24 T_{z}+54\right) p_{z}^{2}+\left(192 T_{z}^{2}-1080 T_{z}+729\right) p_{z}+512 T_{z}^{3}-432 T_{z}^{2}
$$

To determine the number of the solutions to equation (6), we only need to find the zero of the function:

$$
f\left(p_{Z}, T_{Z}\right)=0
$$

since the relationship:

$$
\frac{n^{6}}{729 p_{z}^{4}}
$$

is always higher than zero in the area under consideration. This stems from the fact that both $n$ (the amount of gas moles) is always higher than zero, and also $p_{z}>0$. If we assume that $n=0$, then the analysed environment in which we consider the process would correspond to vacuum, which is outside the scope of our interest. Should we, however, analyse our environment for the $p_{z} \leq 0$ value, it would be pointless from the physical point of view as well, since the object under our consideration is a physical object for which the absolute pressure is always higher than zero. Consequently, $p_{z}$ must be higher than zero.

To solve the cubic equation (10), we must first determine another "discriminant" $\Delta$. Since the form of our equation (9) explicitly implicates that this is a cubic equation due to the variable $p_{Z}$ with the parameter $T_{z}$, we assumed that the "discriminant" for this cubic equation would be denoted as $\Delta_{p}$. The relationship 9 could also be expressed as a cubic equation (12)

$$
f\left(p_{z}, T_{z}\right)=512 T_{z}^{3}+\left(192 p_{z}-432\right) T_{z}^{2}+\left(24 p_{z}^{2}-1080\right) T_{z}+p_{z}\left(p_{z}+27\right)^{2}
$$

due to the variable $T_{Z}$ with the parameter $p_{Z}$. From the mathematical point of view, we will arrive at the same solutions in cases (9), as well as (12). 
To find the solution to equation (10), we must equal the "discriminant" $\Delta_{p}$ for this equation to zero.

$$
\Delta_{p}=\Delta_{p}\left(T_{z}\right)=T_{Z}\left(T_{Z}-1\right)^{3}=0
$$

Thus, we arrive at four solutions, one of which is a triple solution, for which $\Delta_{p}=0$ :

- $T_{z 1}=0-$ is a solution of no physical relevance for us, since it corresponds to the temperature of absolute zero.

- $T_{z 2}=1-$ corresponds to a triple root. When $T_{z}$ takes the value of 1 , the real temperature of the analysed gas $T$ takes the critical value of $T=T_{k r}$.

Having determined the zero of the discriminant $\Delta_{p}(13)$, we can estimate the number of the sought solutions to equation (10) for which $\Delta_{p} T=0$. By adding the $T_{z}$ parameter, in relation to which we will calculate solutions in the set of real numbers, we know that for $T_{z}=1$ and $T_{z}=0$, we are looking for two solutions.

Mathematically, there are three solutions for $T_{z}<0$, which are irrelevant from the physical point of view (and therefore left out of our consideration), since they correspond to a temperature which is below absolute zero.

From a mathematical point of view, equation (10) has in the set of real numbers only one solution for $T_{z}>1$.

For $0<T_{z}<1$, there are three equations (10) in the set of real numbers.

\section{AN ANALYSIS OF THE SOLUTIONS FOR THE DISCRIMINANT $\triangle \mathrm{PT}$}

Going further, if we assume that $T_{Z}=1$, and, by extension, $\Delta_{\mathrm{p}}\left(T_{Z}\right)=0$, we arrive at the following form of equation (9):

$$
f\left(p_{z}, T_{z}\right)=p_{z}^{3}+78 p_{z}^{2}-159 p_{z}+80=\left(p_{z}-1\right)^{2}\left(p_{z}+80\right)=0
$$

The solutions to equation (14) involve the value which is a double root equal to $p_{Z 1}=1$ and $p_{Z 2}=-80$, which is of no interest to us, since, as already mentioned, $p_{Z}$ must be higher than zero.

Conversely, if we assume that $T_{Z}=0$, and, by extension, $\Delta_{\mathrm{p}}\left(T_{Z}\right)=0$, we arrive at the following form of equation (9):

$$
f\left(p_{z}, T_{z}\right)=p_{z}^{3}+54 p_{z}^{2}+729 p_{z}=p_{z}\left(p_{z}+27\right)^{2}=0
$$

The solutions to the above equation (15) are: $p_{Z 1}=0$ and $p_{Z 2}=-27$, which is a double root.

As we know, each cubic equation has three solutions, and not all of these solutions must belong to the set of real numbers. The known solutions based on Cardano's formulas provide the method by which to determine the roots. Regrettably, their formulas provide for operations only on the set of real numbers, with separate calculations for the real and imaginary numbers. Our failed attempts to solve equation (10) based directly on Cardano's formulas prompted us to convert them into a form which would allow us to solve equation (10). To present the method in the clearest possible manner, we defined two coefficients $\alpha$ and $\beta$, whose values belong, in our case, to the set of complex numbers. Thus, the coefficient $\alpha$ was defined as:

$$
\alpha=9 \sqrt[3]{8 \sqrt{\Delta_{p}}-8 T_{z}^{2}-20 T_{z}+1}, \alpha \in \mathbb{Z}
$$

The coefficient $\beta$ was presented as follows

$$
\beta=\frac{81\left(8 T_{Z}+1\right)}{\alpha}, \beta \in \mathbb{Z}
$$

Based on the coefficients so defined, we can present the following solution to equation (10):

$$
\begin{aligned}
& p_{z 1}=\alpha+\beta-8 T_{z}-18 \\
& p_{z 2}=-\frac{1}{2}(\alpha+\beta)-8 T_{z}-18+\frac{\sqrt{3}}{2}(\alpha-\beta) \\
& p_{z 3}=-\frac{1}{2}(\alpha+\beta)-8 T_{z}-18-\frac{\sqrt{3}}{2}(\alpha-\beta)
\end{aligned}
$$

Based on the roots, let us roughly investigate its behaviour towards the parameter $T_{z}$ within the range from zero to infinity, disregarding values below zero, which are physically irrelevant.

The root $p_{z 1}$ within the range $1 \geq T_{z} \geq 0$ has values within the set of real numbers ranging from 0 to 1 . For $T_{z}>1$, its values are within the set of complex numbers. 
For the root $p_{z 2}$, depending on the $T_{z}$ parameter, the values in the range $T_{z}$ under consideration are always negative real numbers, thus being of no interest to us from the physical point of view.

The root $p_{z 3}$ for $1 \geq T_{z} \geq 0$ takes real values from -27 to 1 . For $T_{z}>1 p_{z 3}$ takes values from the set of complex numbers.

The above observations lead us to conclude that due to $\Delta_{p T}=0$, only equation (18) is a solution which a physical sense of interest to us.

An illustration of this reasoning is presented in Figure 2.

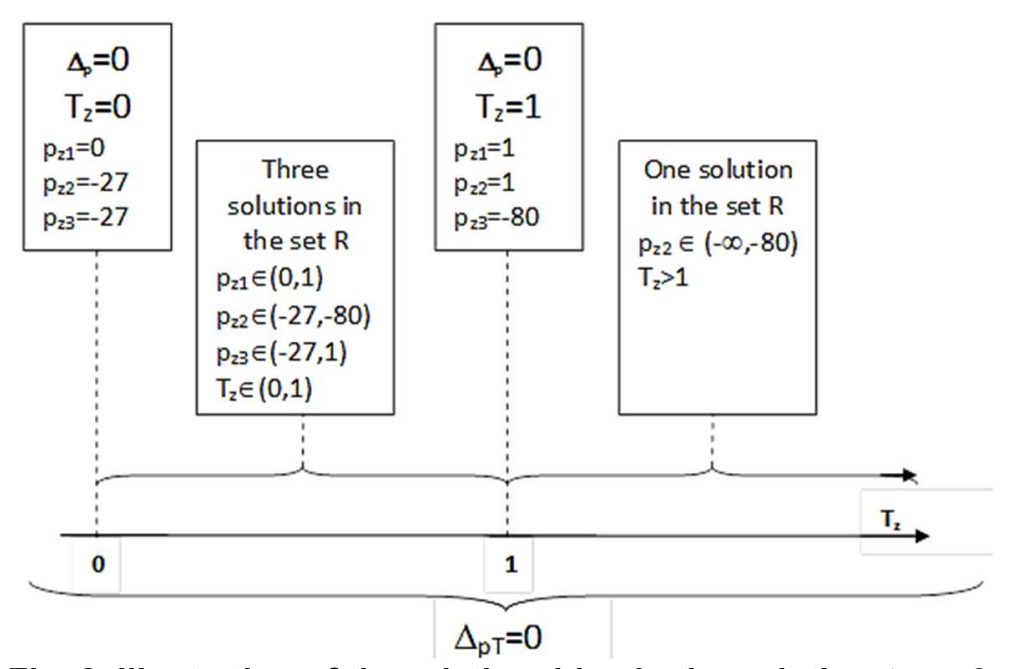

Fig. 2. Illustration of the relationships in the solution $\Delta_{\mathrm{p} T}=0$.

Considering the above, we can proceed to determining, based on equation (6), the volume and, by extension, density, that are of interest to us.

\section{DETERMINING GAS-PHASE DENSITY AS A FUNCTION OF THE DEPTH OF IMMERSION}

After analysing the results, we decided to narrow down our focus to the range of variability in the temperature $T_{z} \geq 1$, corresponding to the real temperature $T \geq T_{k r}$. For most of the gases we analysed (except, for instance, for acetylene $T_{k r}=35.2^{\circ} \mathrm{C}$ and carbon dioxide $T_{k r}=30.98^{\circ} \mathrm{C}$ ) the surrounding air temperature (of sea water) does not fall below the critical temperature for the particular gas, which explains our focus in this study.

If we assume that $T_{1}=1$ and $p_{z}=1$, then equation (6) will produce:

$$
V_{z}^{3}-3 n V_{z}^{2}+3 n^{2} V_{z}-n^{3}=-\left(n-V_{z}\right)^{3}=0 \Rightarrow V_{z}=n \text { (triple) } \Rightarrow V=n V_{k r}
$$

For the values $T_{z}>1$ and $\forall p_{z}>0$, the analysis of the solutions (18)(19)(20) as a function of $T_{z}$ indicates that the discriminant $\Delta_{p} T$ is always higher than zero. It follows, then, that equation (6) has only one solution due to $V_{z}\left(p_{z}, T_{z}\right)$ in the set of real numbers.

The solution due to $V_{z}\left(p_{z}, T_{z}\right)$ for $T_{z} \geq 1$ and $p_{z}>0$ in the set of real numbers is produced as follows.

$$
\begin{gathered}
V_{z}\left(p_{z}, T_{z}\right)=\left\{\begin{array}{c}
n \text { for } T_{z}=1 \wedge p_{z}=1 \\
\frac{n}{9 p_{z}}\left(\gamma+\frac{\left(p_{z}+8 T_{z}\right)\left(p_{z}+8 T_{z}+\gamma\right)-81 p_{z}}{\gamma}\right)
\end{array}\right. \\
\gamma=\sqrt[3]{27\left(p_{z} \sqrt{\Delta_{p T}}+7 p_{z}^{2}+\left(4 T_{z}-27\right) p_{z}+16 T_{z}^{2}\right)+\Delta_{p T}}
\end{gathered}
$$

For $T_{z}<1$, we do not solve the equation because of its considerable complexity and space constraints.

USING THE DERIVED RELATIONSHIPS DESCRIBING CHANGES IN GAS-PHASE DENSITY TO DETERMINE THE AVERAGE DENSITY OF THE TRANSPORT MODULE

The operation of the autonomous transport module, as mentioned in the introduction, is based on a very simple concept involving variations in the module's average density $d_{m}$. If $d_{m}$ is higher than the surrounding water density $d_{H 2 O}$, the module sinks. Conversely, if this density is 
low, the module surfaces. Module density is changed by regulating the amount of water in the transport module. The energy needed for this process is generated by the chemical reaction in which the volume is changing along with the decomposition of substrates into products. The maximum weight that the transport module is capable of carrying to the surface depends on the weight of water that can be displaced from the module when the module achieves equilibrium after reaching the target depth.

The starting point for our discussion, as already mentioned, is determining $d_{m}$ described as the relationship

$$
d_{m}=\frac{m_{K}+m_{z e}+m_{H 2 O}}{V_{m}}
$$

where:

$V_{m}$ is the volume of water displaced from the area occupied by the autonomous transport module,

$m_{K}$ is the mass whose value is independent of the module immersion depth (e.g. structure weight, etc.),

$m_{z e}$ is the mass of the substances which provide the energy for the module surfacing process, $m_{\mathrm{H} 2 \mathrm{O}}$ is the mass of the ballast tank filling the transport module.

In accordance with the law of conservation of mass, in an isolated system $m_{z e}$ must be constant. As this mass is the source of energy for the process of sea-bottom transport, it must undergo certain transformations (e.g. phase transformations). This can be illustrated as equation (24):

where:

$$
m_{z e}=m_{s}(t, p)+m_{p}(t, p)+m_{p g}(t, p)+m_{b g}(t, p)
$$

$m_{s}(t, p)$ is the mass of solid substrates changing while the module is working, with no variations in density;

$m_{p}(t, p)$ is the mass of solid or liquid products of the reaction which shows almost no variations in density occur while the module is working;

$m_{p g}(t, p)$ is the mass of gas products of the substrate-decomposition reaction whose density varies as a function of parameters $t$ and $p$;

$\mathrm{mbg}_{\mathrm{b}}(\mathrm{t}, \mathrm{p})$ is the mass of the transport module's gas buffer whose density varies as a function of parameters $p, t$ and which is necessary for the transport module to work properly.

In this context, the parameter $p$ corresponds to the pressure at a particular depth. The $t$ parameter corresponds to the relative time that passes from the moment the transport module starts to immerse, throughout the process of its immersion, loading and surfacing.

Let us consider again the volume $V_{m}$ - which is an invariable quantity illustrating the volume of the transport module. Taking into account the processes occurring while the transport module is working, we can express this volume as:

where:

$$
V_{m}=V_{k}+V_{z e}(t, p)+V_{H 2 O}(t)
$$

$V_{k}$ is the volume independent of the transport module's depth and immersion; e.g. the volume of the module's structure;

$V_{z e}(t, p)$ is the volume of the source of energy with a mass of $m_{z e}$; due to the changes occurring during the transport process, it is a function of $t$ and $p$;

$V_{\mathrm{H} 2 \mathrm{O}}(t)$ is the volume of the ballast tank filling the transport module and a function of time $t$.

Since $V_{m}$ and $V_{k}$ are in this context invariable quantities, in order for equation (25) to be satisfied, the following relationship must exist:

$$
\frac{\partial V_{z e}(t, p)}{\partial t}+\frac{\partial V_{H 2}(t)}{\partial t}=0
$$

This simply means that a change in the volume $V_{z e}$ must be set off by a change in the volume $V_{H 2 O}$. Therefore, a change in the density $d_{m}$ as a function of the relative time $t$ can be expressed as

$$
d_{m}(t)=\frac{m_{K}+m_{z e}+m_{H 2 O}(t)}{V_{m}}
$$


By extension, we can determine the value of the function $d_{m}(t)$ if we know how the volume of the energy source $V_{z e}(t, p)$ changes over time. To this end, it is necessary to apply the derived relationship (22).

\section{CONCLUSIONS}

In our previous studies of sea-bottom transport, we used discrete calculus. However, this method has a weakness in that it requires us to work from a starting point, and then to verify whether this assumption is correct. In the following steps, we correct this assumption and then repeat the procedure.

By being able to describe a phenomenon continuously in the form of a clearly defined function, we can simulate the process in question using different parameter values. This way, we can select the optimum course of the analysed process, as well as determine derivatives, at any point and with different parameters, to be able to understand how they impact on the process. For example, for a certain point under consideration, we can determine whether it would be better for the achievement of a particular objective to increase the process temperature or to select a different course of the process.

The function describing the process of sea-bottom transport which is of prime interest to us is the one which describes variations in the transport-module's density. Due to the incompressibility or low compressibility of solids and liquids as compared to gases, variations in the average density of the module are described depending largely on gas-phase density, as clearly demonstrated by our equations (26) and (27). Hence, we devoted a major portion of this article to the analytical derivation of variations in gas-phase density as a function of the substance amount $n$, the temperature $T$ and the pressure $p$, allowing us, in accord with relationship (5), to determine the average density of the transport module. Of course, if we are dealing with a mixture of gases, our analysis of the processes should take account of Dalton's law.

In our analysis of the function $V=f(n, p, T)$, we narrowed down our focus to temperature variability in a range equal to or higher than the critical temperature, with no constraints as to the other two parameters. Our article does not provide, however, solutions to $T \in\left(0, T_{k r}\right)$. Even though we have these solutions, they are too complex, so we continue working on developing a method to simplify and present them in a clearer form. We will try to publish the results of these works in the near future.

\section{ACKNOWLEDGMENTS}

This article was written within Statutes Research AGH, No. 11.11.100.005

\section{REFERENCES}

Berry R.S., Rice S.A., Ross J. (2000), Physical chemistry, Oxford University Press, pp 298-306 Bronsztejn I.N., Siemiendiajew K.A. (2000), Matematyka. Poradnik encyklopedyczny, Wydawnictwo Naukowe PWN, Warszawa.

Depowski, S., Kotliński R., Rühle E., Szamałek K. (1998), Surowce mineralne mórz i oceanów, Wydawnictwo Naukowe Scholar, Warszawa.

Filipek W., Broda K. (2016), Theoretical foundation of the implementation of controlled pyrotechnical reactions as an energy source for transportation from the sea bed, Scientific Journals of the Maritime University of Szczecin, 48 (120), 117-124.

Filipek W., Broda K. (2017), The Theoretical Basis of the Concept of Using the Controlled Pyrotechnical Reaction Method as an Energy Source in Transportation from the Sea Bed, TransNav the International Journal on Marine Navigation and Safety of Sea Transportation, Vol.11, No. 4, 653-659.

http://petrowiki.org/images/7/76/Vol1_Page_225_Image_0001.png [Accesed 8 Feb. 2018]

Karlic S. (1984), Zarys górnictwa morskiego, Wydawnictwo „Śląsk”, Katowice

Mizerski W. (2013), Tablice chemiczne, Grupa Wydawnicza Adamantan s.c., Warszawa

Nickalls RWD. (1993), A new approach to solving the cubic: Cardan's solution revealed, The Mathematical Gazette (1993); 77 (November), $354-359$ (jstor) www.nickalls.org/dick/papers/maths/cubic1993.pdf 
Sharma, R. (2017), Deep-Sea Mining: Resource Potential, Technical and Environmental Considerations, Springer International Publishing AG, Cham

Sobota J. et. al. (2005), Systemy i technologie wydobycia konkrecji z dna oceanów. Zeszyty Naukowe Akademii Rolniczej we Wrocławiu nr 521, Monografie XLIII, Seria: Współczesne problemy inżynierii środowiska. Wrocław

Smith E.B. (1990), Podstawy termodynamiki chemicznej, Państwowe Wydawnictwo Naukowe, Warszawa

Smith J.M. et al. (2005), Introduction to Chemical Engineering Thermodynamics, Seventh Edition. McGraw Hill. ISBN 0-07-310445-0

Xiang H.W. (2005). The Corresponding States Principle and Its Practice: Thermodynamic, Transport and Surface Properties of Fluids. Elsevier. ISBN 0-444-52062-7.

SPC (2013), Deep Sea Minerals: Sea-Floor Massive Sulphides, a physical, biological, environmental, and technical review. Baker, E., and Beaudoin, Y. (Eds.) Vol. 1A, Secretariat of the Pacific Community, ISBN 978-82-7701-119-6, Available from: http://gsd.spc.int/dsm/public/files/meetings/ TrainingWorkshop4/UNEP_vol1A.pdf [Accesed 12 July 2015] 